\title{
A rare case of cerebellar and lung tuberculosis coexistence which presenting intracranial mass symptoms
}

\author{
Mükremin $\mathrm{Er}^{1^{*}}$, Ayşe Şule Ateş ${ }^{1}$, Osama Abuzaina ${ }^{2}, \mathrm{H}$. Canan Hasanoğlu ${ }^{2}$ \\ ${ }^{1}$ Pulmonary Diseases Department, Ataturk Training and Research Hospital, Ankara, Turkey \\ ${ }^{2}$ Pulmonary Diseases Department, Yoldirm Beyazıt University, Ankara, Turkey
}

\begin{abstract}
Tuberculosis continues to be a problem in the entire world and can be confused with other diseases, when presented extrapulmonary involvement. Cerebellar tuberculoma may present with symptoms of intracranial mass.

A 53-years-old male patient was admitted with complaints of dizziness, nausea and vomiting. Cranial magnetic resonance imaging was observed mass in the posterior fossa and the patient was operated. Pathology of the lesion was reported as necrotizing granuloma. Patient diagnosed with tuberculosis meningoencephalitis and treated standard four drug anti tuberculosis regimen plus methylprednisolone. The symptoms of the patient were completely disappeared during treatment. Follow-up magnetic resonance imaging which taken 6 months after onset of the treatment shows cranial tuberculoma sequelae in the cerebellum.

Central nervous system tuberculosis may be difficult to diagnose as it is an infrequent entity that can easily mimic an intracranial mass. Especially in tuberculosis endemic areas, in the patients who presenting with symptoms of intracranial lesion, tuberculosis diagnosis should be considered.
\end{abstract}

Key Words: Tuberculosis, extra-pulmonary, intracranial mass, cerebellar tuberculoma

\section{Introduction}

Tuberculosis is still a common disease in Turkey, and diagnosing tuberculosis can sometimes be challenging as its extrapulmonary manifestations can be confused with several other conditions. Central nervous system (CNS) involvement of tuberculosis is rare and encountered in almost $5-10 \%$ of patients with active tuberculosis. While the most common clinical presentation is meningoencephalitis, cerebellar involvement accounts for 3-4\% of these cases (1). However, diagnosing cerebellar tuberculosis can be very difficult, particularly in patients without a diagnosis of primary tuberculosis.

Tuberculoma is the most common manifestation in parenchymal cases. Although less often, the disease can also manifest as cerebritis or encephalopathy. Majority of cerebellar tuberculoma cases in the literature were reported in children, but some cases were also reported in patients aged up to 45 years (24). The rare case described here involves a patient who was operated with the preliminary diagnosis of a cerebellum tumor, but later discovered to have cerebellar tuberculosis accompanied by an atypical picture of pulmonary tuberculosis.

\section{Case report}

A 53-years-old male patient without a previously known disease referred to an external center with complaints of dizziness, nausea and vomiting. Imaging assessments showed a mass in the posterior fossa and the patient was operated. Pathological examination showed necrotizing granulomatous event and cyclophosphamide therapy was initiated with the preliminary diagnosis of sarcoidosis. The patient subsequently developed a clinical picture of meningoencephalitis and referred to infectious diseases clinic of our hospital. The patient was consulted to us at this point and a thorax computerized tomography (CT) was performed, which showed conglomerate lymph nodes at mediastinal and hilar localizations, pleural effusion and multiple centrilobular nodules with lobulated contours (Figure 1).

This case report was presented as discussion poster at the "Turkish Thoracic Society 18th Annual Congress" at Antalya, 1-5.April.2015. (PS095)

*Corresponding Author: Mukremin Er, M.D. Ataturk Training and Research Hospital, Department of Pulmonary Diseases, 06800 Bilkent, Ankara, Turkey Phone: +90 (312) 29125 25, Fax: +90 (312) 29127 26, E-mail: mukreminer@hotmail.com 


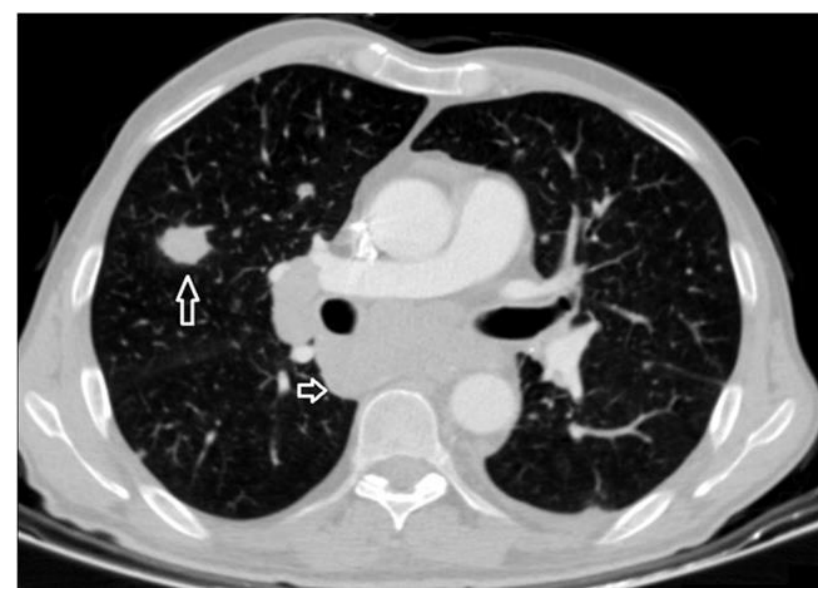

Fig. 1. Thorax CT scan showed centrilobular nodules with lobulated contours and conglomerate lymph nodes at mediastinal and hilar localization.

When the patient admitted to our department his general medical status was normal and he was partially immobilized because of dizziness. Patient's medical history did not include any other condition except smoking history of 30 pack-years. Family history showed that the patient's uncle had a history of tuberculosis. His tuberculin skin test (TST) was measured $17 \mathrm{~mm}$, and initial laboratory findings were as follows: hemoglobin: $133 \mathrm{~g} / \mathrm{L}$ (normal range: 135$180 \mathrm{~g} / \mathrm{L}$ ), white blood cell count: $7.46 \times 10^{9} / \mathrm{L}$ (normal range: $5-14.5 \times 10^{9} / \mathrm{L}$ ), platelet count: $307 \times 10^{9} / \mathrm{L}$ (normal range: $150-400 \times 10^{9} / \mathrm{L}$ ), Creactive protein: $160 \mathrm{mg} / \mathrm{L}$ (normal range: $0-5 \mathrm{mg} / \mathrm{L}$ ), urea: $200 \mathrm{~g} / \mathrm{L}$ (normal range: 10-48 g/L), creatinine: 7 $\mathrm{g} / \mathrm{L}$ (normal range: $<1.2 \mathrm{~g} / \mathrm{L}$ ), aspartat transaminase: $93 \mathrm{U} / \mathrm{L}$ (normal range: $0-40 \mathrm{U} / \mathrm{L}$ ), alanin transaminase: $172 \mathrm{U} / \mathrm{L}$ (normal range: 0-41 U/L). Additional tests were performed to rule out other granulomatous diseases, and serum angiotensin converting enzyme (ACE) level was found $29 \mathrm{U} / \mathrm{L}$ (normal range: 8-52 U/L), brucella hemagglutination and tularemia tests were negative. Microbial growth was not seen in the culture media of blood, pleural fluid and cerebro-spinal fluid (CSF) samples. Lymphocyte predominance was noted in the CSF. Positron emission tomography (PET), which was performed to rule out any primary malignancy or metastases, showed mild standard uptake value (SUV) in the cervical, mediastinal, hilar and abdominal lymph nodes and in the parenchymal multiple nodular lesions. Despite insufficient cellularity, pathologic examination of the lymph node biopsy samples obtained by endobronchial ultrasound

(EBUS) did not demonstrate malignant cells. Contrast magnetic resonance imaging (MRI) of the brain showed enhanced contrast uptake in the pial surfaces of the infratentorial and supratentorial regions (Figure 2).

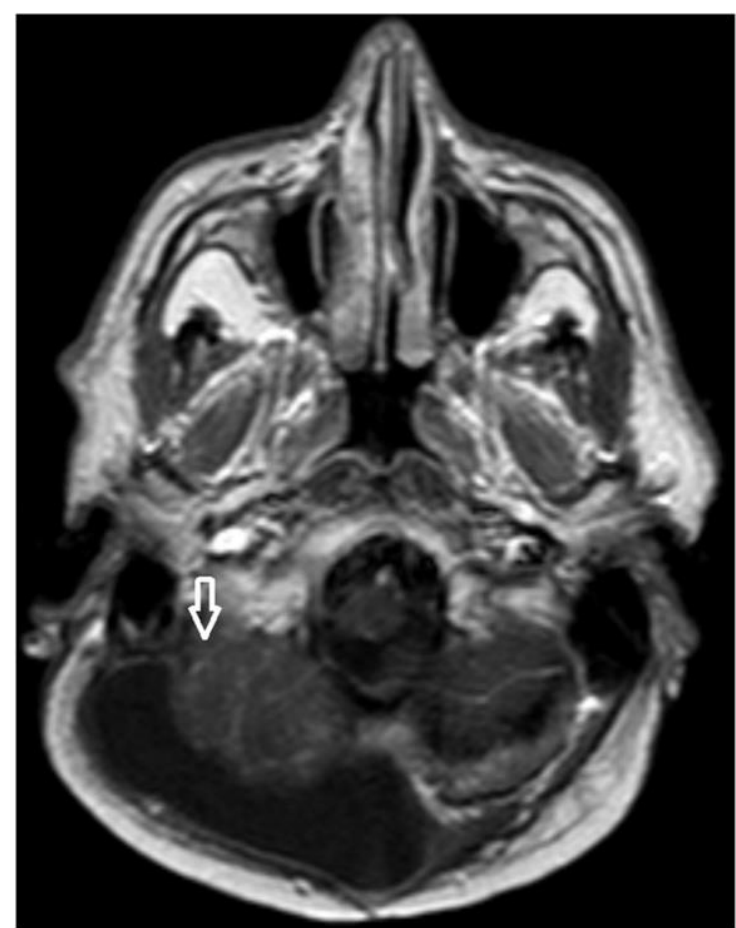

Fig. 2. Magnetic resonance imaging (MRI) of the brain showed enhanced contrast uptake in the pial surfaces of the infratentorial and supratentorial regions.

Standard four-drug tuberculosis treatment regimen was initiated with the preliminary diagnoses of pulmonary and cerebellar tuberculosis, and methylprednisolone therapy was added with the preliminary diagnosis of meningoencephalitis. The patient's symptoms and clinical findings markedly improved during follow-up period, and he was discharged to continue tuberculosis treatment on an outpatient basis. Following completion of the 9months treatment for tuberculosis, methylprednisolone dose of the patient was also reduced. The patient showed marked clinical improvement. Follow-up thorax CT scan demonstrated shrinkage of the mediastinal and hilar lymph nodes and absence of pleural effusion, while the other findings were stable (Figure 3).

Follow-up cranial MRI after 9 months antituberculose treatment showed multiple tuberculomas in the right cerebellar hemisphere and the vermian region (Figure 4).

\section{Discussion}

Central nervous system tuberculosis more frequently occurs in the presence of conditions such as HIV infection, malnutrition, immunosuppression and alcoholism (5). The case presented here involved a 53 years-old male patient who had no known cause of immunosuppression. 


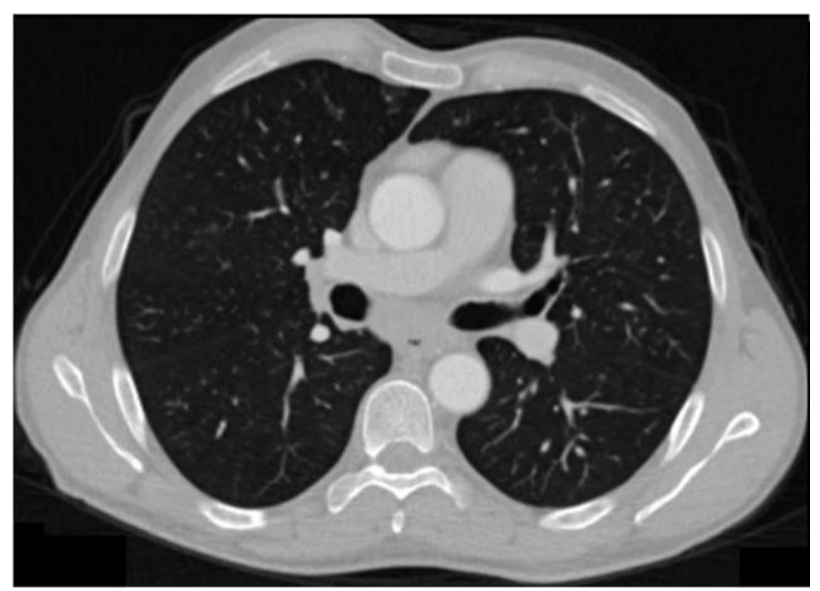

Fig. 3. Follow-up thorax CT scan shows disappearance of the parenchymal lesion which existed previously and shrinkage of the mediastinal and hilar lymph nodes.

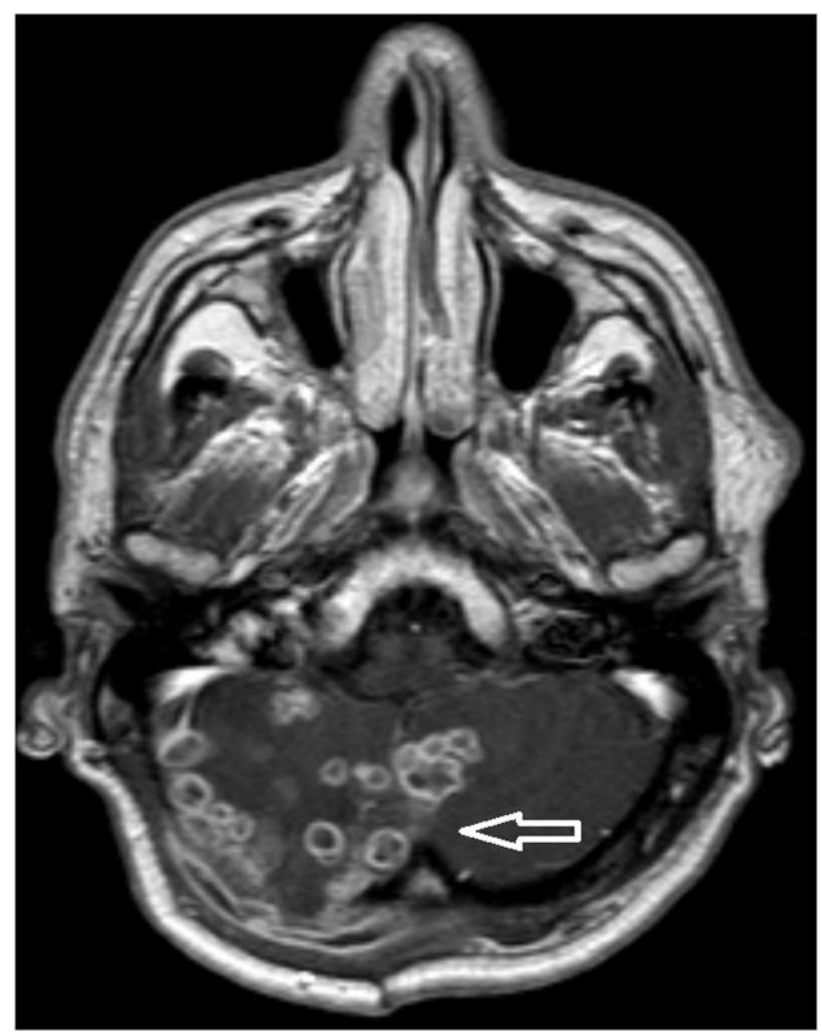

Fig. 4. Follow-up cranial MRI scan shows multiple tuberculomas with rim-enhanced borders in the right cerebellar hemisphere and the vermian region.

Laboratory findings alone aren't helpful in diagnosing cerebellar tuberculosis generally. For this reason, radiological imaging plays key roles in diagnosis, follow-up and identification of the complications. Radiological findings of parenchymal tuberculomas may appear as lesions with enhanced peripheral rim or nodular contrast, that result in mild to moderate peripheral edema and mass effect $(6,7)$. On the other hand, primary masses, are generally cerebellar hemangioblastomas, medulloblastomas or glioblastomas. As tuberculomas do not have typical radiological findings, they cannot be differentiated from the other masses by using radiological methods. The case presented here had been operated in an external center with the preliminary diagnosis of a posterior fossa mass. The patient developed a clinical picture of meningoencephalitis later. Moreover, CT and MRI scans obtained during follow-up showed that centrally-necrotic, tuberculoma-like mass appearances with peripheral contrast reappeared in the operated region and in the cerebellum.

Diagnosing central nervous system tuberculosis requires the diagnosis of primary disease to be supported by cytological examination of the CSF, microbiological evaluations (EZN staining), demonstration of antibodies or antigens in CSF, and radiological findings $(8,9)$. Golden standard for diagnosis is the presence of bacteria in pathological examination or demonstration of the caseification necrosis (10). In the case presented here, tuberculosis diagnosis was supported by the pathological findings that revealed necrotizing granulomatous reaction, as well as the presence of other factors such as mediastinal and hilar conglomerate lymph nodes, a TST result of $17 \mathrm{~mm}$, findings of meningoencephalitis, and a family history of tuberculosis. Our diagnosis was further confirmed by disappearance of the lung lesions and appearance of typical tuberculomas in the cerebellum after the patient had been treated for tuberculosis.

Central nervous system tuberculosis may be difficult to diagnose as it is an infrequent entity that can easily mimic an intracranial mass. In regions where tuberculosis is an endemic disease, clinical suspicion constitutes an important step of the diagnostic pathway, along with laboratory and imaging findings. Moreover, treatment response can retrospectively confirm the diagnosis in cases where a definitive diagnosis cannot be established.

Disclosure: Authors have no conflict of interests, and the work was not supported or funded by any drug company.

Acknowledgements: We thank to H. Canan Hasanoğlu Prof. M.D. and Ömer Faruk Ateş M.D for valuable contributions during preparation of the manuscript.

\section{References}

1. Caws M, Wilson SM, Clough C, Drobniewski F. Role of IS6110-targeted PCR, culture, biochemical, clinical, and immunological criteria for diagnosis of tuberculous meningitis. J Clin Microbiol 2000; 38: 3150-3155.

2. Click ES, Moonan PK, Winston CA, Cowan LS, Oeltmann JE. Relationship between 
Mycobacterium tuberculosis phylogenetic lineage and clinical site of tuberculosis. Clin Infect Dis 2012; 54: 211-219.

3. Farinha NJ, Razali KA, Holzel H, Morgan G, Novelli VM. Tuberculosis of the central nervous system in children: a 20-year survey. J Infect 2000; 41: 61-68.

4. Grimud-Ayina M, Fari O, Lortholary O, et al. Neuro-meningeal tuberculosis in northeastern suburbs of Paris. Nineteen cases. Ann Med Interne 2002; 153: 75-81.

5. Gurjar HK, Joshua SP, Agrawal D, Mahapatra AK. Large pontine tubercular abscess treated surgically. Br J Neurosurg 2013; 27: 134-136.

6. Mazodier K, Bernit E, Faure V, et al. Central nervous tuberculosis in patient's non-HIV: seven case reports. Rev Med Interne 2003; 24: 78-85.
7. Phypers M, Harris T, Power C. CNS tuberculosis: a longitudinal analysis of epidemiological and clinical features. Int J Tuberc Lung Dis 2006; 10: 99-103.

8. Sonmez G, Ozturk E, Sildiroglu HO, et al. MRI findings of intracranial tuberculomas. Clin Imaging 2008; 32: 88-92.

9. Taşçı C, Gündoğan A, Tehli Ö, et al. A Case of Cerebral-Cerebellar Tuberculosis with Presented Syncope. Turkiye Klinikleri Arch Lung 2015; 16: 43-47.

10. Wang JT, Hung CC, Sheng WH, et al. Prognosis of tuberculosis meningitis in adults in the era of modern antituberculous chemotherapy. J Microbiol Immunol Infect 2002; 35: 215-222. 